\title{
Editorial
}

Marketing communication "gets" DAM. Short and to the point, this issue of the Journal of Digital Asset Management examines how reuse and reexpression of digital media and related workflow automation speed up the marketing communications process.

By way of introducing DAM for marketing communications, let's start with brief history of "marcom" told in internet time.

In the now distant past of ten or so years ago, large- and medium-sized companies spent a great deal of time and money developing a marketing strategy, sharing that strategy with their creative partners (mostly advertising agencies) and watching that strategy undergo a lengthy and tortuous transformation into delivered brandmarketing messages (ink on dead trees and red, green, and blue dots on electronic displays).

Then, almost overnight, the internet and the web erupted, surprising many firms. The headlong rush to create a web presence entailed taking a lot of short cuts; in particular, this resulted in the creation of single-purposed use asis content (what many of us called "crap") and the need for content (er, crap) management systems. In the shadows of crap management, DAM skulked and lurked. Digital assets and the need to manage these assets would take several years to emerge as a core discipline for what marketers today call "multichannel brandmarketing communications."

Fast-forward to 2005. Even today there remain among us individuals who cannot differentiate assets from crap. So the world turns.

Many brand-marketers today see their network of creative and production partners as nodes of an increasingly all-digital supply-chain. With this new vision came profound insights about how DAM can transform a loose-knit network of creative and production shops into strategic suppliers of a marcom supply-chain; suppliers are now subject to more disciplined procurement and compensation processes.

This brings us to our first paper in which Skiff Wager, our resident DAM-IT guru, explains the "Create, ingest, manage, distribute and measure" or CIMDM building block model of an enterprise asset management strategy, and emphasizes the central role that metadata plays in managing a DAM-driven business.

The second paper in this issue is "Strategic sourcing of marketing content" by Hanna-Maija Nyberg of TeliaSonera, one of Europe's largest telecommunication firms. She explains how DAM and related workflow automation tools drove an EU-wide consolidation of creative and production partners.

The baton is taken up by Simon Myers of Adgistics in his paper, "How the application of DAM-enabled MRM will reshape the marketing communications process (and industry)." He links DAM with other spokes in the wheel of marketing operations management: budgeting, planning, campaign management, sourcing and metrics for marketing communications.

Robert Watts drills down into the heart of DAM, explaining how brand marketing and a global network of resellers needed speedy access to high-resolution artwork and photography. In a nutshell, DAM + web = image portals.

One of the most onerous aspects of global brand management is tackled by Geert Wirtjes and Debbie Wirtjes-Lewis, namely delivering final packaging artwork in support of worldwide product launches. In much the same vein as Hanna-Maija Nyberg, the authors explain how Philips Domestic Appliances use asset-activity analysis and user logs to derive high-level business intelligence about their marcom activities. This underscores a key theme for DAM: activity-data analysis can provide closed-loop statistical-quality controls of complex processes.

Tony McCarthy explains how DAM has become to transform the business models and practices of advertising agencies. In particular, he discusses how DAM enables agencies to provide new services and earn incremental revenue from doing so.

TranTech's Mark Wells delivers a reinterpretation of the value proposition for DAM in the government sector, highlighting both the enormity and diversity of the public sector market. 


\section{Lest not we forget}

DAM first took root in media, entertainment, and publishing - industries that create and transform digital assets into cold hard cash: video games, TV shows, comic books, and magazines. In this issue of the Journal of DAM, we hear from a few pioneers.

Tony Blake of the CM Group introduces the idea of an online archive, one of the first real destinations in the journey of DAM.

Arkemedia's Neil McLaren takes on the other side of the DAM universe - video and multimedia assets - in his paper "The pervasive nature of DAM across a multi-media organization". The author makes a persuasive case that DAM addresses both print and electronic media.

The project management and strategies of video-centric DAM is taken on by Guillaume Tourneur of VVV in "Media asset management - Analysis and methodology overview". He makes a simple and powerful case that technology does not a solution make; rather, it's all process. A great reminder for us all: he who has the best process not only wins the day, but wins the war.

In the final paper in this issue, Karen Fitchpatrick of Scripps Network - home of Home \& Garden Television and several other popular cable network channels - explains how they use DAM in marketing digital inventories of TV programs to advertisers and how traditional software applications and DAM merge, creating an integrated process automation platform.

Finally, please email us with your thoughts and feedback about the Journal of DAM, sharing with us what you like, like to read more about, or your ideas for contributing an article.

Cheers!

\section{Michael Moon Managing Editor}

